\title{
RESEARCH ON THE DECISION MAKING SUPPORT FUNCTION USING THE MARKOV PROPERTY
}

\author{
Akinori MINAZUKI, Susumu KUNIFUJI \\ Graduate School of Knowledge Science \\ Japan Advanced Institute of Science and Technology \\ 1-1,Asahhidai, Tatsunokuchi-cho, Nomi-gun \\ Ishikawa 923-1292, Japan \\ E-mail; minazuki@jaist.ac.jp, kuni@jaist.ac.jp
}

Keywords: Markov property, Decision support, Car Navigation System, Expected utility

Summary: The driver sometimes is not satisfied by the route chosen by the Car Navigation System. Therefore, the function and the concept of the current Car Navigation System were examined. It can be said that the driver's subjectivity can be changed based on objective conditions. There are three main results. 1. Derive the objective element, factoring in predicted conditions. 2. Based on a conditional node, make predictions which will make a judgment about the subjective element. 3. Example all possible route and at the conditional node make a judgment about the subjective element based on the weight of all the conditions.

\section{Introduction}

The background of the research was obtained from a practical example of decision-making problems. It is a system rapidly spreading throughout Europe, America and Japan. The system can be described a functional part of the car, guiding the driver to the desired destination by the displaying road information. This is called a Car Navigation System in Japan. When this is used, the drivers depend on the map information shown on the display. Based on the input given by the driver, the Car Navigation System shows the most efficient route based on time and distance. Generally, drivers do not drive the most direct route to the destination point. Usually when driving, even if a person preplans the route, he will have a desire to make impulsive stops along the way. Depending on the driver, he might even stop for brief rest to enjoy the view or spend some time on a leisure activity. These types of stops enhances the driving pleasure and are determined subjectively based on the individual driver. The driver can input desired leisure spots into the Car Navigation System. This input causes the Car Navigation System to plan desired stops along the final destination point. However after driving, the driver sometimes is not satisfied by the route chosen by the Car Navigation System. The dissatisfaction is caused by the system's inability to anticipate the driver's immediate need. For example, based on the driver's pretend stop points, the system may direct the driver to a tennis court, but by the time of arrival, it could be raining or the road driven may be congested, slowly the drive. In these cases, the drive to the courts would have been time wasted. The driver's desired stop point is subjective input while the change in weather change or traffic patterns are objective elements. The destination decided is influenced by both of these factors. Even if these are many objective elements, they are transitional so events that may influence can be predicted. Therefore, the function and the concept of the current Car Navigation System were examined. Although the system's function can guide the driver to the desired destination points, but it cannot forecast changes in driving conditions. In the future, there is a prospect for a new function of Car Navigation System based on model designed using an AHP. 


\section{Does the shortest route satisfy the decision-makers .}

The present Car Navigation System is based on an algorithm that drives through all desired stop points regardless of the driver's ultimate aim. The present Car Navigation System uses a VICS function. It guides by route based on an objective element, traffic concentration. The VICS Car Navigation System defaults to a previously decided if the traffic concentration decreases. Occasionally this route will not take the driver in the desired direction. This action is similar to the effect described in Planned and Situational Actions. That is, the VICS Car Navigation System, which is about to return to the previously decided route, might not correspond completely to the driver's aim. When the system makes a detour, the driver may have a new aim, but the VICS system's function will still point out the originally intended route.

For this point, the guarantee of the shortest route breaks down. This type of situation occurs most frequently when the driver desires a change due to altered weather conditions.

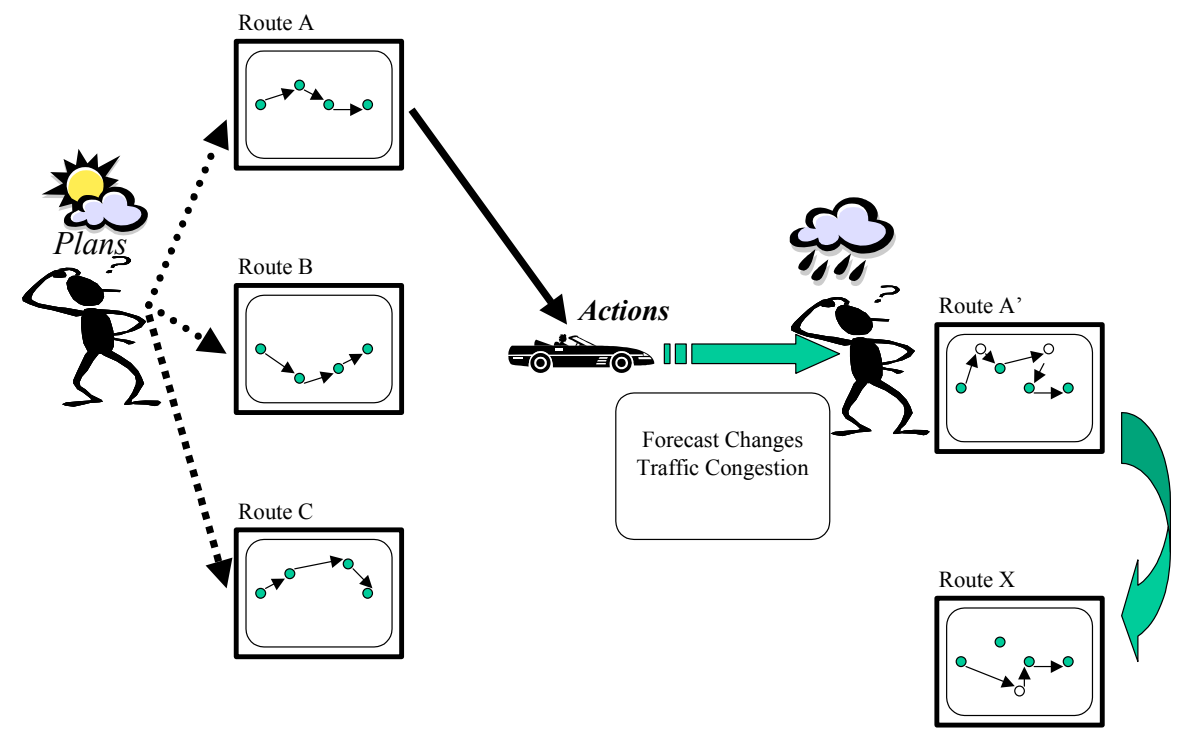

Fig.1 Guarantee of the shortest route plan

\section{Purpose and features of the research}

\subsection{Purpose of the research}

That's why it can be said that the driver's subjectivity can be changed based on objective conditions. The aim is to have the ability to make decisions based on both elements, so the ability to make predictions based on current conditions has to be created. This diagram shows the influence of the objective criteria on the subjective criteria. In order to derive these figures, a decision making model with Markov characteristics was designed.

In order to create the model, three ideals were initially proposed.

1. Derive the objective element, factoring in predicted conditions.

2. Based on a conditional node, make predictions which will make a judgment about the subjective element.

3. Example all possible route and at the conditional node make a judgment about the subjective element based on the weight of all the conditions. 


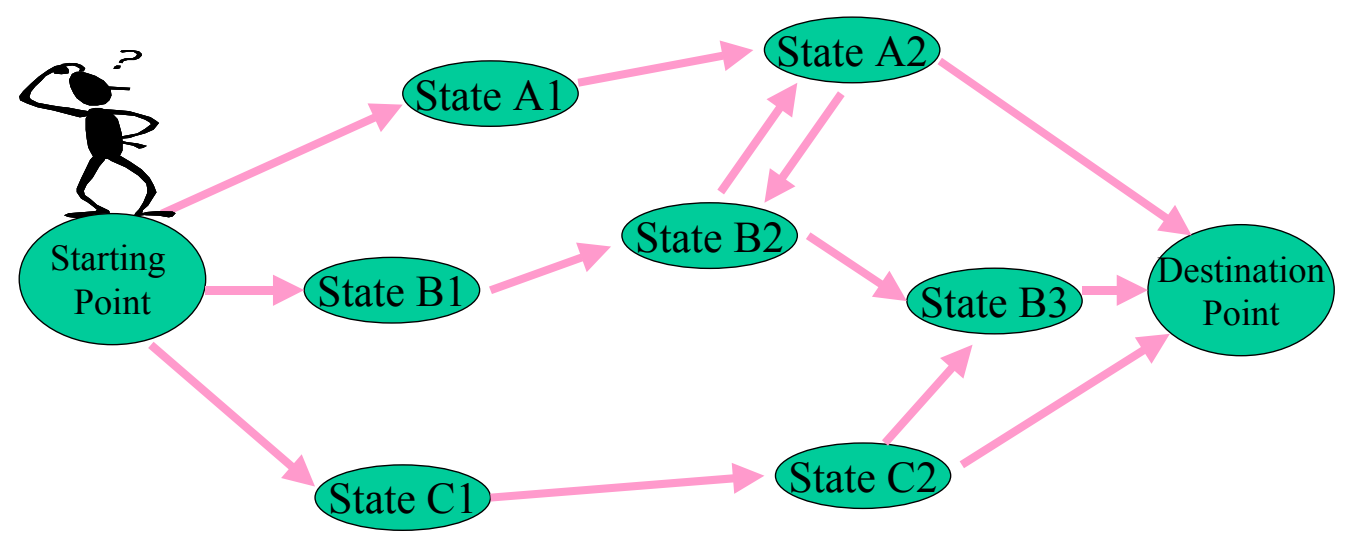

Fig.2 State transition chart of a decision-making situation

\subsection{Features of the research}

There are the 3 features of this research.

1. At the point of decision-making, an objective element with the Markov property is extracted.

2. At the point of decision-making, the subjective and objective influence evaluations are assimilated.

3. Assimilate a ratio scale by pairing comparisons based on the value of the probability scale determined by the Markov property. (Construction of New Decision Process)

The system this research proposes takes into considerate the decision maker's preference and the choices available. Based on a pair of linked functions choose the best route while deciding on alternatives. Based on the AHP and Markov process if will contain a Preference/Choice algorithm. This is called MDS with AHP. The preference function calculates the objective element of the decision- making process. The choice function calculates the subjective element of the decision- making process. Deciding the best route from both functions does not necessarily mean connecting the states on the shortest node. The decision-maker (driver) does not always choose a route that is the shortest route. People look for enjoyment in their chosen routes. Of course, a route will be avoided if there is an element which will prevent enjoyment.

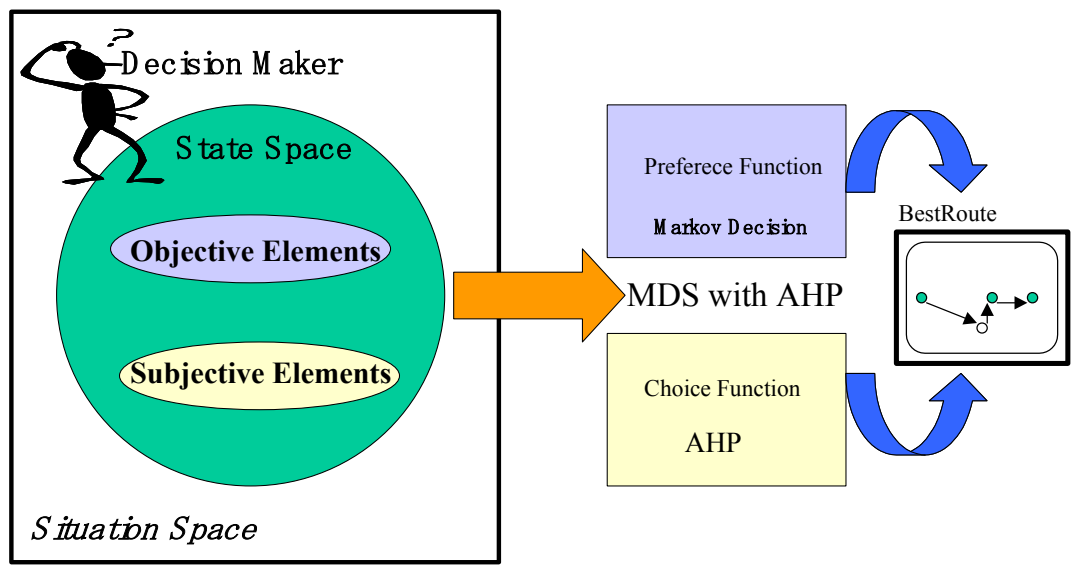

Fig.3 MDS with AHP 


\section{Definition of the Markov property in decision making process: A Markov decision} process formulation follows

The general framework: Our framework is as follows.

(1) A system occupies one of a finite set of states at the beginning of each of a set of time units, which we label $t=1,2, ., .$, . moving forward from some time origin.

$$
\begin{aligned}
& \text { Decision epochs }: t=\{1,2, \ldots ., N\}, \quad N \leq \infty \\
& \text { States }: S=\{0,1,2, \ldots \ldots, M\} . \\
& \text { Actions }:\{0,1,2, \ldots \ldots, M-s\} .
\end{aligned}
$$

(2) We designate the state set by $X_{j}$ to denote the possible dependence on $t$ for each value of $t$. We write $p(j \mid i) \equiv P\left\{X_{n}=j \mid X_{0}=i_{0}, \ldots, X_{n-1}=i_{n-1}\right\}=P\left\{X_{n}=j \mid X_{n-1}=i_{n-1}\right\}$ and refer to it as a transition probability. We call the matrix. $P$ with $(i, j)$ th component $p(j \mid i)$, the transition probability matrix or transition matrix.

$$
\begin{aligned}
& p_{i j}=P\left\{X_{n}=j \mid X_{n-1}=i\right\}, \quad 1 \leq i, \quad j \leq N \\
& 0 \leq p_{i j} \leq 1, \quad \sum_{j=1}^{N} p_{i j}=1 \\
& p=\left[\begin{array}{cccc}
p_{11} & p_{12} & \cdots & p_{1 N} \\
p_{21} & p_{22} & \cdots & p_{2 N} \\
\vdots & \vdots & & \vdots \\
p_{N 1} & p_{N 2} & \cdots & p_{N N}
\end{array}\right]
\end{aligned}
$$

(3) In a stationary Markov chain, we denote the $m$-step transition probability by $p^{m}(j \mid i)=P\left\{X_{n+m}=j \mid X_{n}=i\right\}$. As a consequence of repeated application of the law of total probabilities, $p^{m}(j \mid i)$ is an element of the matrix $p_{i j}^{(m)}$, the $m$ th power of the matrix $P$.

$$
p_{i j}^{(m)}=\sum_{k=1}^{N} p_{i k}^{(m-1)} p_{k j}
$$

(4) The random variable $X_{t}$ will denote the state at the beginning of time unite $t$, and realized values will be denoted by $X_{j}(n)$ for each value of $t$.

$$
X_{j}(n)=\sum_{i=1}^{N} P(n) P^{(n)}
$$

(5) For each state $X_{t}=X_{j}$ there is a feasible action space $X_{j}^{\alpha}$, where $X_{j}^{\alpha}$ is finite for each value of $t$.

(6) The system evolves as a no stationary Markov chain with transition probability matrices. 


\section{The expected utility}

\subsection{Deriving the expected utility}

This will not only be effective in Car Navigation Systems but the Markov property can also be used in other decision making processes. In this decision making model, the expected utility is derived from two influence degrees in the state space: an objective influence weights and a subjective influence weights. The relative criticality of the objective expected utility and subjective expected utility are determined for the final route of the expected utility based on preferred.

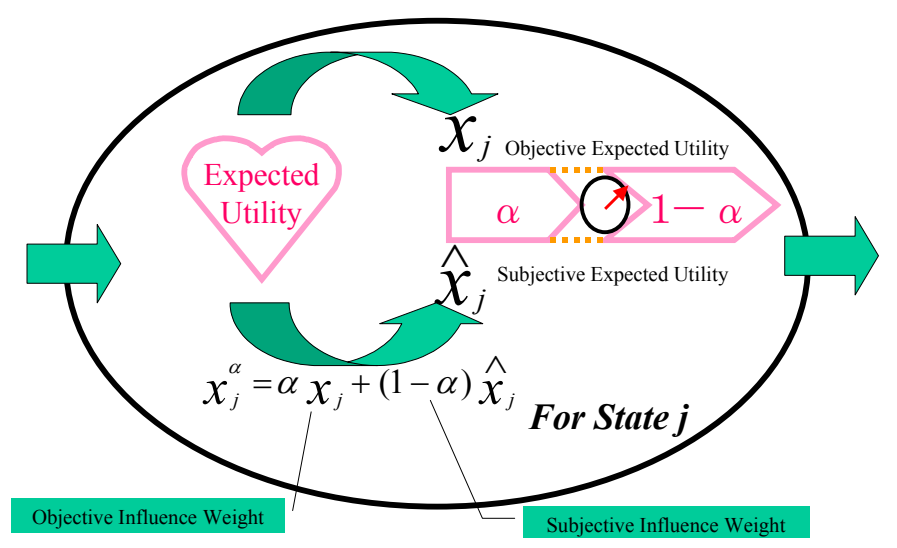

Fig.4 Subjective influence and objective influence in the state

(1) The expected utility of a state point

$$
\begin{aligned}
& X_{j}^{\alpha}=\alpha X_{j}+(1-\alpha) \hat{X}_{j} \quad 0 \leq \alpha \leq 1 \\
& \alpha=\left[\begin{array}{ll}
X_{j 1} & X_{j 2}
\end{array}\right]\left[\begin{array}{c}
\hat{X}_{j 1} \\
\hat{X}_{j 2}
\end{array}\right]
\end{aligned}
$$

(2) Expected utilities of each route

$$
\begin{gathered}
W_{r}^{\alpha}=\alpha \min \left(\sum_{i=1}^{N} X_{j}^{\alpha}\right)_{r}+(1-\alpha) \max \left(\sum_{i=1}^{N} \hat{X}_{j}^{\alpha}\right)_{r}, \quad 0 \leq \alpha \leq 1, r=A, B, C \cdots \\
\alpha=\min \left[\begin{array}{c}
\left(\sum_{i=1}^{N} X_{j}^{\alpha}\right)_{A} \\
\left(\sum_{i=1}^{N} X_{j}^{\alpha}\right)_{B} \\
\left(\sum_{i=1}^{N} X_{j}^{\alpha}\right)_{C}
\end{array}\right] \max \left[\begin{array}{c}
\left(\sum_{i=1}^{N} \hat{X_{j}^{\alpha}}\right)_{A} \\
\left(\sum_{i=1}^{N} \hat{X_{j}^{\alpha}}\right)_{B} \\
\left(\sum_{i=1}^{N} \hat{X_{j}^{\alpha}}\right)_{C}
\end{array}\right]
\end{gathered}
$$

\subsection{Analysis of the expected utility}

To calculate a relative criticality, some representative points were decided. Thus, the decision-maker's chooses from among the Risk Avoiding type, the Neutral type, and the Dangerous Acknowledgment type when choosing a final route. The $\alpha$ shows the level of pessimism and optimism the 
decision-maker has. We calculate again by using the $\alpha$, and obtain the best route. It is unlikely that the order of best route obtained from both functions of the system is the same.

\section{Practical application of the model}

\subsection{Driving examples}

Let us take a leisurely drive as an applied example.

At the time of departure, the drivers decide on playing tennis and hiking in the mountains. For the drive route, we have to take into consideration the weather condition, road congestion, and the seasonal aspects. We cannot avoid these considerations. Therefore, we can actively assimilate the transition of objective elements on the driving route with the subjective demand element, and decide the best route.

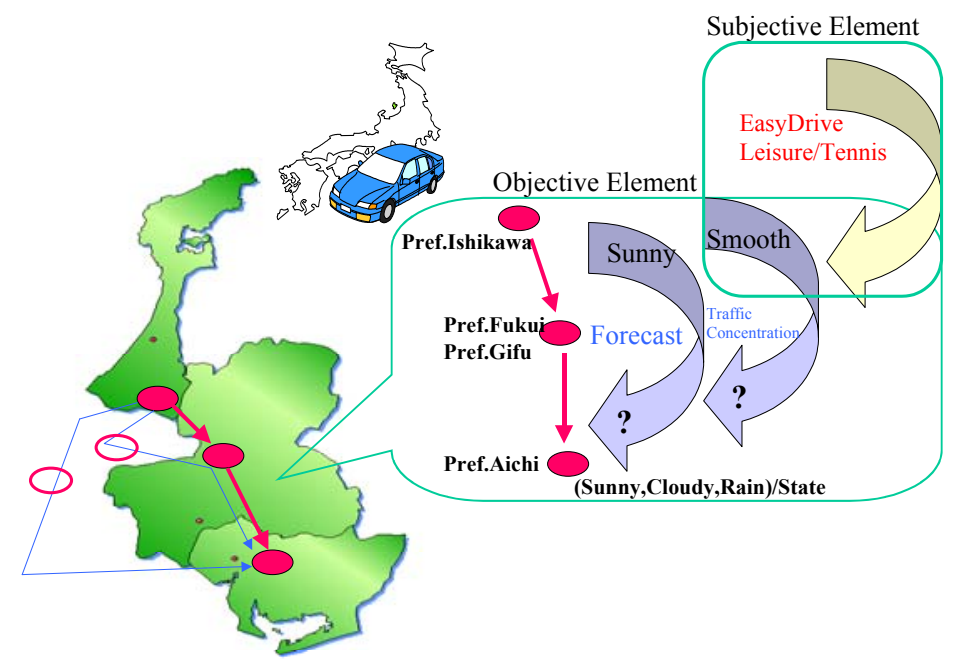

Fig.5 Driving through the central part of Japan

\subsection{Calculation of the Markov elements}

Table1. Analysis result 


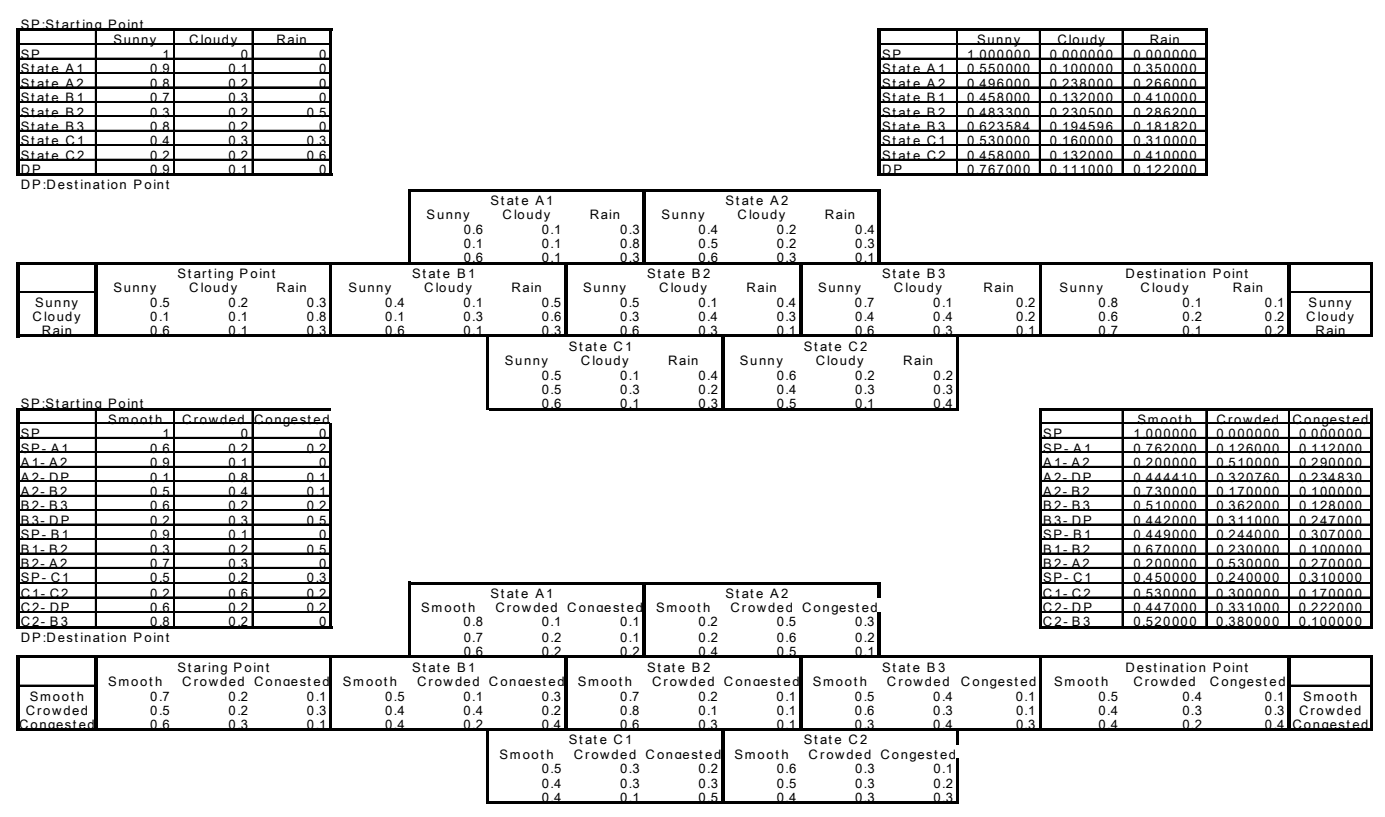

Table2. Analysis result using $\alpha$ :

Calculation results for each route
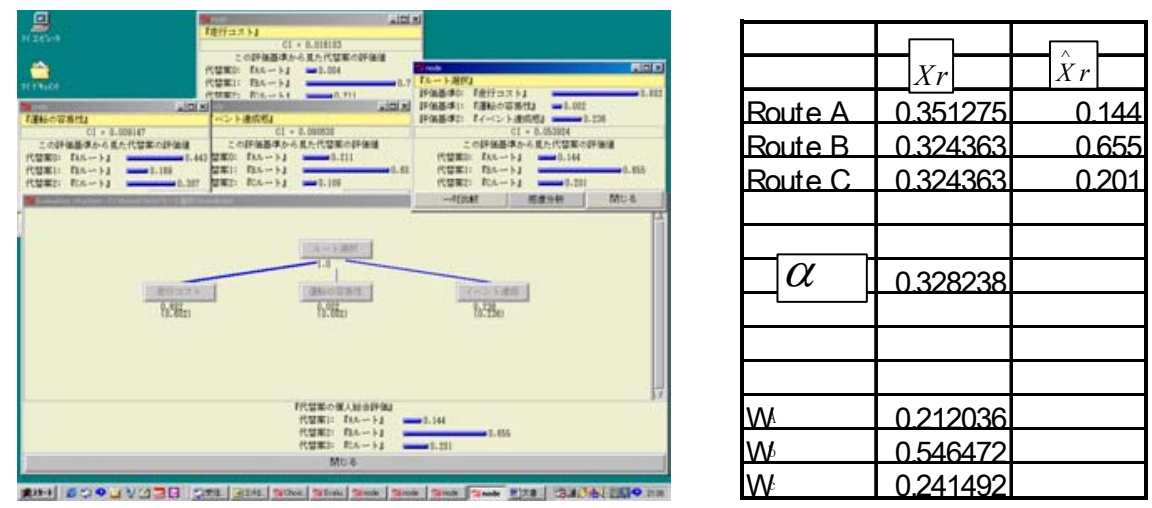

Fig.6 Markov interface AHP

\section{Conclusion}

There are three main results.

1. In the preference process and the choice process of the decision-making action, the probability scale by the Markov property was applied to the evaluation based on the ratio scale constructed by the AHP.

2. The expected utility in each state of the decision making process was assimilated, and it weighed the expected utility of the objective item against the subjective item.

3. In the route path selection, the evaluation was done considering the evaluation based on the objective expected utility and the influence of the state transition between routes to other states. 
The research of ITS (Intelligent Transport Systems) is worldwide, and it is a new transport system which is comprised of an advanced information and telecommunications network for users, roads and vehicles. In addition, the Japan's road policy also collaborate in the promotion of ITS. And ITS contributes much to solving problems such as traffic accidents and congestions. ITS user services are research by allocating users for each of the nine different development fields in accordance with individual users needs and conditions under which these needs arise. In this period, which is the initial stage of ITS, traffic information will be distributed via VICS and similar systems which have already begun service. Traffic congestion information and optimum routes will be displayed on in-vehicle navigation system so that the driver can achieve pleasant travel including travel time reduction, and wish our model to contribute partially of the ITS function in the future.

\section{References}

Suchman, Lucy A. (1987), Plans and Situated Actions, Cambridge University Press.

Puterman, Martin L. (1994), Markov decision Processes-Discrete Stochastic Dynamic Programming, A Wiley-Interscience Publication.

White, Douglas.John. (1992), Markov decision processes, John Wiley \& Sons Publications.

Morimura, Hidenori. (1991), OR Library18-Markov Analysis, Nichikagiren Publications.

Tenmoku, Kenji. (2000)," Navigation System and Optimal Route," Journal of Japan. Operations Research, Vol.45, No.7, 312-318 